\title{
Multifunctional Carbon Fiber Sensors: The Effect of Anisotropic Electrical Conductivity
}

\author{
Norbert Forintos, Tamas Sarkadi, Csanad Ors Boros, Tibor Czigany
}

\begin{abstract}
The multifunctional application of carbon fiber reinforced polymer composites (CFRP) can be affected by the anisotropic electrical conductivity of the carbon fiber because it differs in the longitudinal and the transverse direction of the fiber. In our article, we measured the transverse resistivity of the reinforcing carbon fiber bundle in different conditions: when the fibers were dry, when they were impregnated with non-crosslinked resin, and in a crosslinked matrix. We have shown that in the case of dry and impregnated fibers, a compressive force acting on the fiber bundle has a significant effect on the transverse resistance, while when crosslinked, this effect is negligible. We developed a finite element model (FEM) to investigate the area around the electrodes and the current distribution in the fiber bundles. We

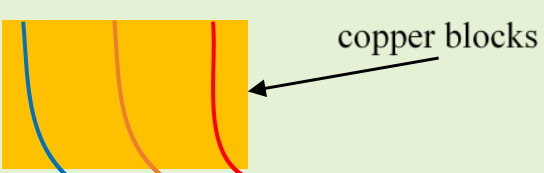
have shown that due to the pressure dependence of the transverse resistance and the significant difference in length and transverse conductivity, the current density distribution in the cross-section around the current electrode will not be homogeneous, leading to inaccuracies in sensory applications. We have shown by finite element simulation that the current electrode and voltage measurement electrode can be optimized, which was verified experimentally.
\end{abstract}

Index Terms - multifunctional sensor, carbon fiber, electric resistance, impregnation, finite element analysis

\section{1Introduction}

$\mathrm{C}$ ARBON fiber reinforced polymer composites (CFRPs) are increasingly used in various industries (the automotive, energy, and other sectors) and their use is projected to increase further [1]. One of the reasons for this is that with CFRPs have high strength, load-adjustable rigidity, they can be manufactured and machined easily, and have excellent surface quality, while their density is low $[2,3]$. Another reason for their popularity is the steps taken towards recyclability. From the production of CFRP to the end of its lifetime, a large amount of waste is generated, which must be recycled - it reduces the environmental impact on the one hand and recovers costly raw material (carbon fiber) on the other. Due to ongoing research on recycling, it is becoming more attractive for many industries to make components from primary and recycled carbon fiber [4]. Another process that helps spread carbon fiber composites is the emergence of multifunctional materials, which act as structural materials and perform a secondary function (sensing, heating, actuation) [5-7]. We have previously written an overview article on the multifunctional use of carbon fibers and the possible secondary functions, where we also systematized

This work was supported by the OTKA (K 120592) and NVKP (NVKP_16-1-2016-0046) projects of the National Research, Development, and Innovation Office (NKFIH), and by the NRDI Fund (TKP2020 NC,Grant No. BME-NC; and TKP2020 IES,Grant No. BMEIE-NAT) based on the charter of bolster issued by the NRDI Office under the auspices of the Ministry for Innovation and Technology.

N. Forintos is with Department of Polymer Engineering, Faculty of Mechanical Engineering, Budapest University of Technology and Economics, 1111 Budapest, Muegyetem rkp. 3. and MTA-BME Research Group for Composite Science and Technology, 1111 Budapest, Muegyetem rkp. 3. the raw materials and auxiliary materials necessary for the application [8].

Among the most important multifunctional applications are sensory applications, where the effects on the CFRP component can be monitored by continuously measuring the electrical resistance of the carbon fiber. Hasan et al. [9] placed a carbon fiber roving in a fiberglass and polypropylene fiber composite and then measured the carbon fiber's resistance during a tensile test. From the change in resistance, they were able to determine the degree of deformation. Luan et al. [10] placed a carbon fiber roving on a test specimen made by additive manufacturing. They varied the load location and measured the resistance of the carbon fiber while performing a three-point bending test on the specimen. From the change in resistance, they were able to calculate the load on the specimen. Pinto et al. [11] measured the change in fiber resistance in a carbon fiber reinforced epoxy matrix specimen during a bending test. They found good correlation between resistance change and deformation. Eddib and Chung [12] measured a CFRP specimen's capacity along its length using alternating current. From the increase in capacity measured along the thickness, they were able to deduce the

T. Sarkadi is with Department of Atomic Physics, Faculty of Natural Sciences, Budapest University of Technology and Economics, 1111 Budapest, Budafoki street 8.

O. C. Boros is with Department of Atomic Physics, Faculty of Natural Sciences, Budapest University of Technology and Economics, 1111 Budapest, Budafoki street 8.

T. Czigany is with Department of Polymer Engineering, Faculty of Mechanical Engineering, Budapest University of Technology and Economics, 1111 Budapest, Muegyetem rkp. 3. and MTA-BME Research Group for Composite Science and Technology, 1111 Budapest, Muegyetem rkp. 3. (e-mail: czigany@eik.bme.hu). 
damage to the specimen, and from the capacity measured in the plane of the specimen, they were able to determine the amount of damage and the distance from the electrode. The electrical resistance of carbon fiber also depends on the temperature change; its resistance decreases as the temperature increases. After measuring the temperature-resistance relationship, that is, calibrating the sensor, the resistance of carbon fiber reinforcement can provide important information about the crosslinking of the matrix, and it can be used to control the heat treatment even during production and to monitor the heat load on the finished product. [13].

Electrically insulating materials, such as fiberglassreinforced polymers, can also be modified to provide electrical current-based condition monitoring. Alexopoulos et al. [14] used a carbon nanotube-filled epoxy resin for fiberglassreinforced specimens, making the original insulating composite conducting. By measuring the electrical resistance of the material they produced, they could determine failure due to overload or external influence. The conductivity of the resin can also be improved if the composite is reinforced with a conductive fiber, such as a woven carbon fiber fabric. Kumar et al. [15] used a conductive polymer, polyaniline, as the matrix material. In their research, they wanted to reduce the harmful effects of a lightning strike on aircraft. In their experiments, they found that in a composite with better conductivity, the current of lightning is distributed faster at the point of impact, and the Joule heat load is smaller. As a result, after the lightning strike, the specimen retained a large percentage of its original stiffness. Song et al. [16] coated flexible wool yarns with silver nanowires and formed a cross-ply arrangement. They used the overlapping are as pressure sensor, as the electric resistance of the connecting plies varied with pressure. They found good sensibility and reliability, which makes the modified wool a potential material for wearable electronics.

The examples showed that carbon fiber can be used as a sensor under laboratory conditions, but this requires knowledge of the electrical properties (such as conductivity) of the individual carbon fibers and the carbon fiber bundles and fabrics. The conductivity of fiber bundles is strongly anisotropic. The current flows parallel to the fibers by several orders of magnitude more easily than perpendicular to the fiber bundles. For this reason, the contacts of the electrodes connected to the fiber bundles need to be designed carefully.

Owston [17] used a Wheatstone bridge to measure the resistance of carbon fiber. He calculated a specific resistance of $5.8-31 \mu \Omega \mathrm{m}$ in different carbon fiber samples. Safarova and Grégr [18] measured the resistance of a polyacrylonitrile (PAN) and a tar-based dry carbon fiber bundle in a two-point arrangement. Tar-based carbon fibers had better conductivity due to the more perfect graphite lattice. Their specific resistance ranged from 5 to $6.6 \mu \Omega \mathrm{m}$, while the specific resistance of PAN-based carbon fibers ranged from 14.5 to $20.5 \mu \Omega \mathrm{m}$.

Athanasopoulos and Kostopoulos [19] measured the resistance of dry unidirectional (UD) carbon fiber fabrics in the fiber direction and perpendicular to the fiber direction. They calculated a resistivity of $31.99 \mu \Omega \mathrm{m}$ in the fiber direction and $26300 \mu \Omega \mathrm{m}$ perpendicular to the fiber direction. They explained the large difference with the fact that a current perpendicular to the fibers can only flow through the random fiber-to-fiber contacts.
Zimney et al. [20] investigated the current in an anisotropic material during four-wire resistance measurement using a finite element model. They found that due to the different longitudinal and transverse conductivities, the current density in the material was not uniform, so the apparent change in crosssection must be taken into account when the resistance is measured. They also showed that the voltage measuring electrode significantly influences the change in current density due to its better conductivity.

When impregnated with a non-crosslinked resin or in a crosslinked matrix, the carbon fiber bundle can ideally be considered as resistors connected in parallel, assuming perfect impregnation. However, due to the imperfect production, there are also fiber-to-fiber contacts, so electrical current can be conducted in a direction different from the fiber's orientation; thus, the measured resistance may differ from the calculated resistance [21].

We aim to use the reinforcing carbon fibers as a sensor by measuring their resistance. By continuous measurement of the fiber bundle's resistance, continuous condition monitoring can be performed, which provides information about the deformation and failure of the structure (for example, automotive parts). However, the measured resistance strongly depends on the location of the current input and the measuring conditions (e.g., contact quality). The purpose of this paper is to determine the path of the current flowing in the carbon fiber near the electrodes and further in the bundle. To achieve this,

- we developed a tool with which we measured the transverse resistance of the carbon fibers as a function of compressive force;

- we prepared a finite element model of a four-wire resistance measurement layout based on the transverse and longitudinal resistivity of the carbon fiber, which we validated by measurement;

- we optimized the geometry of the current electrodes based on the model, and made the distribution of current density more uniform;

As the media around the reinforcing carbon fiber changes during the composite structure production, we measured the resistance of dry fibers, fibers impregnated with noncrosslinked resin, and fibers in a crosslinked matrix, just as it would change during an actual manufacturing process.

\section{EXPERIMENTAL}

In our experiment, we used PAN-based Sigrafil C T245.0/270-E100 (SGL Carbon SE, Germany) carbon fibers: the number of filaments was 24 thousand, density was $1.81 \mathrm{~g} / \mathrm{cm}^{3}$, fiber diameter was $6.9 \mu \mathrm{m}$, tensile strength was $5 \mathrm{GPa}$, the tensile modulus of elasticity was $270 \mathrm{GPa}$, elongation at break was $1.9 \%$, and resistivity was $14 \mu \Omega \mathrm{m}$ [22]. The fibers are coated with sizing to improve the adhesion between the fibers and the matrix. In our experiment, we didn't remove the sizing as we wanted to examine the fibers' conductivity properties as they would be used in industrial applications. We called the fibers with sizing but without resin "dry fibers".

To determine the transverse resistance of the carbon fiber bundle, we designed two prototype tools and manufactured them from polylactide acid (PLA) by fused deposition molding (FDM) (Fig. 1). The tools' role was to fix the electrical connection required for resistance measurement, ensure the 
geometry of the sample made up of carbon fiber bundles, and load the sample with pressure. The electrical connection (maximum voltage was $50 \mathrm{mV}$ ) between the carbon fiber sample and the data acquisition device (Agilent 34970A, Agilent Technologies, USA) was established with copper blocks (tightening torque was $10 \mathrm{Nm}$ ).

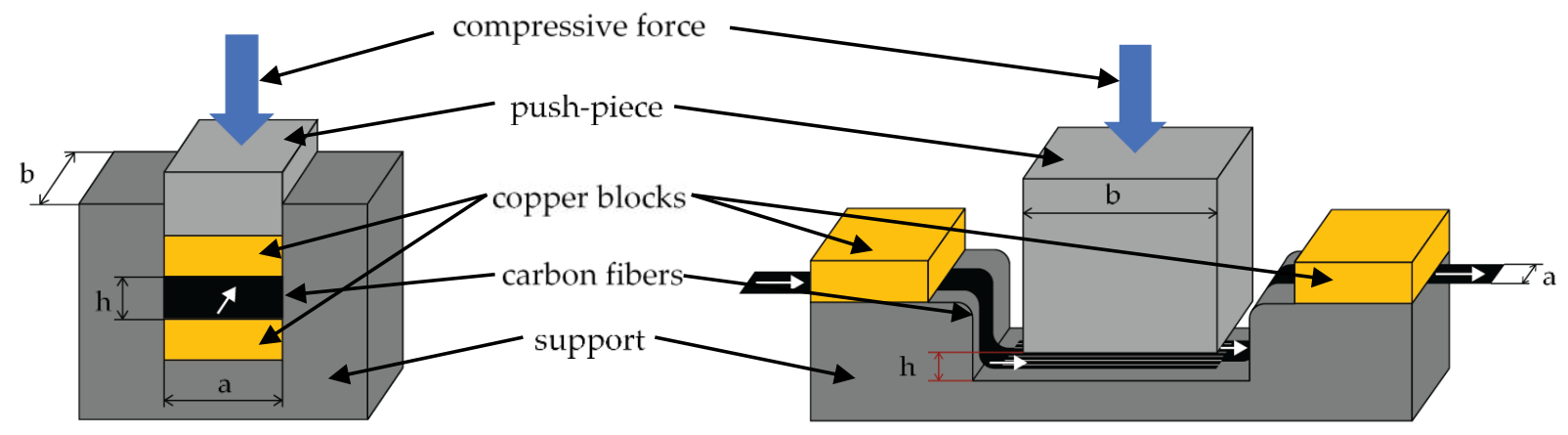

a)

b)

Fig. 1. Schematic model of a tool developed for the dry transverse resistance measurement of a carbon fiber bundle (a) and a longitudinal section of a tool developed for the impregnation and pressure testing of carbon fibers (b). The white arrows show the direction of the fibers

\section{A. Transverse resistance measurement}

We measured the transverse resistance using a two-wire method and a sampling time of $2 \mathrm{~s}$. We placed carbon fiber bundles in the tool made by FDM (Fig. 1 a) and measured the sample's geometry. First, we measured the transverse resistance unloaded, and then placed the tool in a universal testing machine. (Zwick Z250 Zwick, Germany). We applied various compressive pressures $(0.005 ; 0.01 ; 0.02 ; 0.04 ; 0.08 ; 0.16$; $0.32 ; 0.64 ; 0.96 ; 1.28 ; 1.52 \mathrm{MPa})$ to the specimen through the push-piece. The initial values correspond to the order of magnitude of the pressures occurring during manual lamination, while the final values correspond to the order of magnitude of the pressures used during vacuum manufacturing technologies. We measured three samples, and the resistance of the sample was measured three times at each loading force. The loading speed was $2 \mathrm{~N} / \mathrm{min}$, the load holding $(10 \mathrm{~min}$ ) was force-controlled and the unloading speed was $20 \mathrm{~mm} / \mathrm{min}$. From the beginning of the load, the resistance was recorded every two seconds for 5 minutes after the load.

In the tool in Fig. 1 a, we were able to measure the transverse resistance of the dry carbon fibers accurately, due to the location of the copper blocks and the sample. In order to impregnate the fibers with resin, and examine them in the crosslinked matrix, we further improved the arrangement (Fig. 1 b). In this arrangement, we changed the way the current was coupled, but we compensated for this during evaluation. We performed the experiments on carbon fibers impregnated with a non-crosslinked resin (Ipox MR 3016, Ipox Chemicals Kft., Hungary) and also in a crosslinked matrix material (MR 3016 and MH 3124 100:40 mixing ratio, Ipox Chemicals Kft., Hungary). For this, we used the improved tool, which was also manufactured by FDM. Impregnation, crosslinking, and pressure loading in a universal tensile machine can all be performed in this tool (Fig. $1 \mathrm{~b}$ ).

\section{B. Longitudinal resistance measurement}

We measured the longitudinal resistance of a dry carbon fiber bundle in a four-wire arrangement; the measurement length (0$1000 \mathrm{~mm}$ ) was the distance between the voltage measurement electrodes (Fig. 4 a). We measured the resistance of three fiber bundles, three times at each distance.

\section{Finite element modeling}

The conducting phenomena in single carbon fiber and at fiber-fiber contacts were investigated and modeled earlier [17, 23]. In our research, we inspected the macroscopic conducting effects in the transversely isotropic material model described below. We modeled the electric current density within the carbon fiber bundle in Comsol Multiphysics FEM software. The fiber bundle was replaced with a transversely isotropic material model; we set the electrical conductivity parameters according to our measured values. Electrical conductivity was examined in 2D on the geometry of the fiber bundle. The finite element mesh was rectangles, condensed towards the edges and around the copper blocks. (Fig. 2).

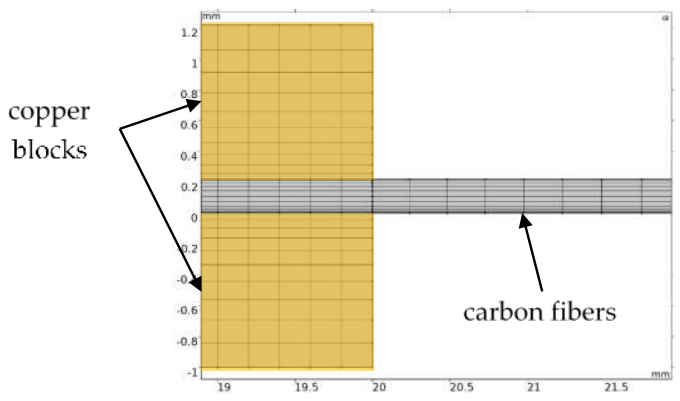

Fig. 2. Finite element model of the area of a copper block electrode

\section{Results AND discussion}

It is important to measure the carbon fibers' resistance accurately when used in sensory applications. To better understand the current distribution in the carbon fiber specimens, we measured the transverse resistance and made a finite element model validated by longitudinal resistance measurement. Using the FEM model, we examined and optimized the environment and geometry of the current electrodes.

\section{A. Transverse resistance}

We started evaluating the results by examining the transverse resistance in the measurement setup shown in Fig. 1. During the test, resistance changed in time after loading, and approached a constant value. This behavior can be explained with the fibers' 
physical location: as the fibers are compressed by the load, more and more contacts are formed between the fibers, thereby reducing the resulting resistance of the sample. We regarded the resistance for a given load as the resistance at the end of the 10minute measurement. We subtracted the resistance of the system $(0.14 \Omega)$, which is the resistance measured on the copper blocks without the fibers. We determined the transverse specific resistance $\left(\rho_{t}\right)$ of the sample from the stabilized resistance $(R)$, the width $(a)$ and length $(b)$ of the sample, and the distance $(h)$ between the copper blocks (1), then plotted it as a function of pressure (Fig. 3).

$\rho_{t}=\frac{R * a * b}{h}$

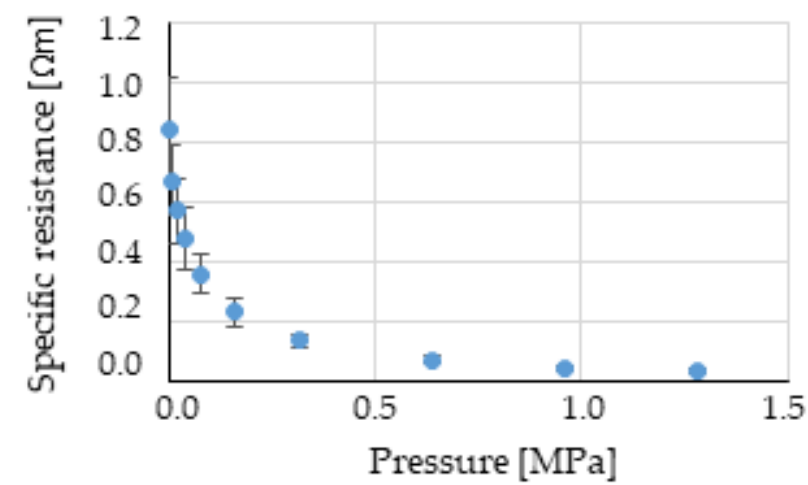

Fig. 3. Transverse specific resistance of carbon fiber bundle as a function of pressure, the average of three specimens

We found that the transverse specific resistance of dry carbon fibers greatly depends on the compressive load. This is important in the electrode area, where resistivity changes as the copper blocks are compressed. Therefore, we examined the surrounding area of the copper blocks by finite element analysis.

\section{B. Finite element modeling}

We modeled a four-wire longitudinal resistance measuring arrangement with the finite element method. For this, we prepared a transversely isotropic material model of the fiber bundle with the measured results of the transverse specific resistance and the catalog data of the longitudinal specific resistance $(14 \mu \Omega \mathrm{m})$ [22] of the carbon fiber bundle. In the case of copper blocks, we assumed that the bundle was compressed by the tightened blocks; therefore, we used the following transverse specific resistance values, which are close to the pressures used and the specific resistances measured in the actual measurement arrangement (Fig. 3): $0.22 \Omega$ m measured at $0.16 \mathrm{MPa}$ near the copper blocks, and $0.54 \Omega \mathrm{m}$ measured at $0.01 \mathrm{MPa}$ further from the blocks (Fig. 4 a). These values were verified by measurement described later in this paragraph.

In the modeled experimental setup, the current was introduced through the copper blocks marked "A" and conducted through the copper blocks marked "D"; we call these current electrodes. Electrodes "B" and "C" are the electrical connections needed to measure the voltage drop; we call these voltage electrodes. It is clear from the finite element results that due to the strong anisotropy of the current in the vicinity of the electrodes, a significant proportion of the current flows in the part of the fiber bundle that is in direct contact with the copper blocks (red arrows) and not in the full cross-section (Fig. 4 a). This uneven current density distribution homogenizes further away from the copper blocks. In the section below the copper blocks, current density is distributed faster due to the excellent conductivity of the copper blocks and compression, while after the copper blocks, it is slower due to the lower transverse conductivity. The FEM results showed that due to the excellent conductivity of the copper blocks, there is a "drain effect" at the voltage electrodes. The essence of the drain effect is that the conductivity of the fiber bundle between the copper blocks of the voltage measuring electrode is significantly lower than the conductivity of the copper blocks. Because of this, a portion of a)

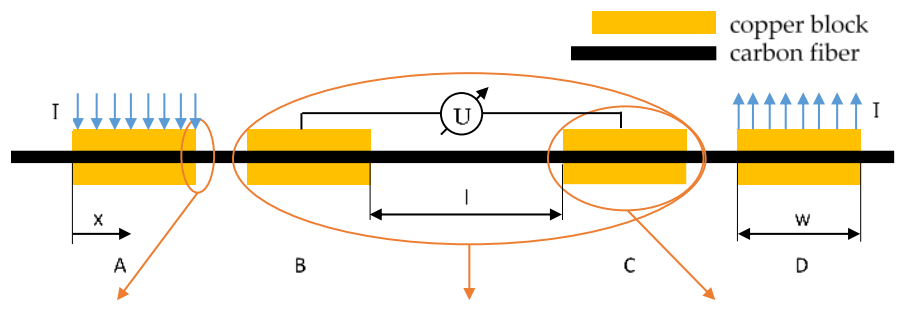

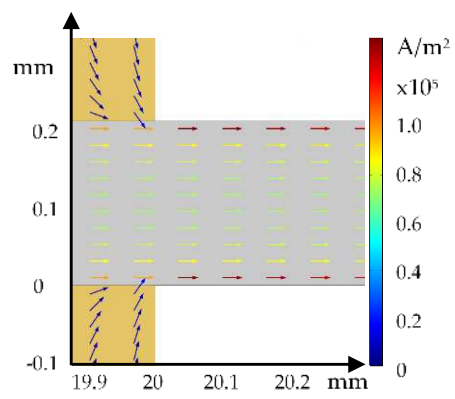

b)

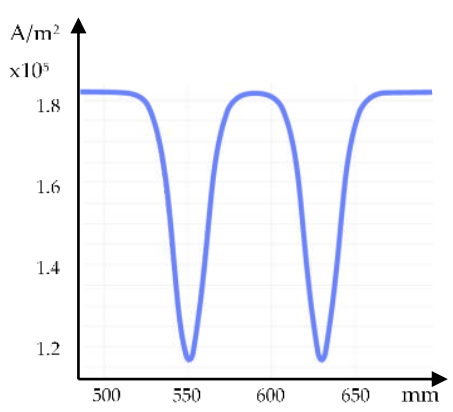

c)

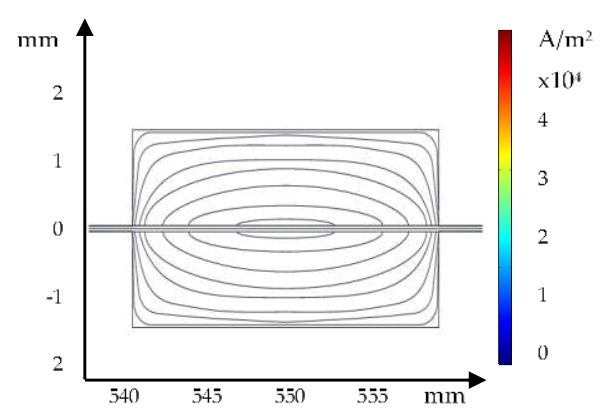

d)

Fig. 4. Schematic diagram of the four-wire resistance measurement layout (a), representation of the current density vectors near the current electrode in the FEM software (b), the longitudinal distribution of current density in the centerline based on the FEM simulation (I=60 mm) (c) and the "current drain" effect of the copper blocks used for voltage measurement, as represented by current lines the in FEM software (d). 
the current passes from the fiber bundle to the copper blocks, where it continues to flow approximately parallel to the fiber bundle. Since the wires of the voltmeter do not conduct significant current from the copper blocks, the current flowing in the copper blocks returns to the fiber bundle on the other side of the copper blocks. This effect also results in an uneven current density distribution along the centerline of the fiber bundle (Fig. 4 c).

The simulation shows that there must be a difference between the specific resistance values given in the catalog and the calculated values when the longitudinal conductivity is determined because the current density is not homogeneous in the section influenced by the voltmeter blocks, and the conductive cross-section cannot be determined accurately. Therefore, in the environment of copper blocks, the simplified formula (2) of calculating resistivity will not be valid.

We experimentally verified the results of the finite element model by measuring longitudinal resistance at different lengths. To determine the longitudinal specific resistance $\left(\rho_{l}\right)$, we calculated the theoretical cross-section from the fiber diameter $(d)$, and the elementary fiber number of the fiber bundle $(n)$. For the calculation of specific resistance, we also used the measured fiber bundle length $(l)(2)$.

$\rho_{l}=\frac{R * n * d^{2} * \pi}{4 l}$

The results showed the expected effect: at small measurement lengths, where the current density is inhomogeneous in the cross-section, we measured a higher specific resistance than expected, which is a consequence of the poorly estimated conductive cross-section and inhomogeneous conduction. We validated our FEM model by comparing the measured and simulated specific resistance values. (Fig. 5).

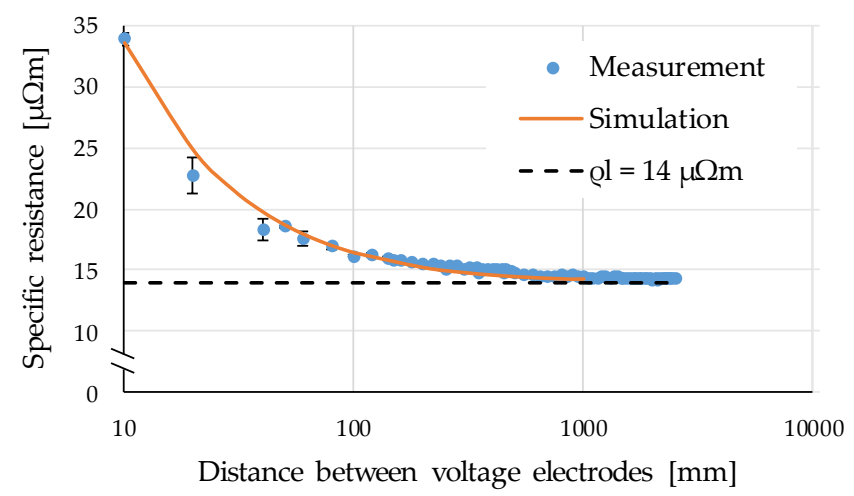

Fig. 5. Validation of the FEM results based on the comparison of measured and calculated specific resistance values

The results of the finite element model simulating the measurement arrangement approximate the measurement results well; this indicates that the finite element model can take into account the average macroscopic effects of the different conduction phenomena occurring in the fiber bundles with sufficient accuracy. However, we did not obtain the expected results because we used the simplified formula (1) to calculate the resistivity. To find the limits of the simplified formula, we further investigated the area of the current and voltage electrodes with the validated FEM.

\section{Optimization of current supply geometry}

Based on the validated results, we investigated whether a more homogeneous current distribution in the carbon fiber bundle at and near the voltage electrodes could be achieved by changing the geometry of the copper blocks. We ran the validated simulation with $\mathrm{w}=10 \mathrm{~mm}$ and $\mathrm{w}=30 \mathrm{~mm}$ widths in addition to the original $\mathrm{w}=20 \mathrm{~mm}$ wide copper blocks (Fig. 6).

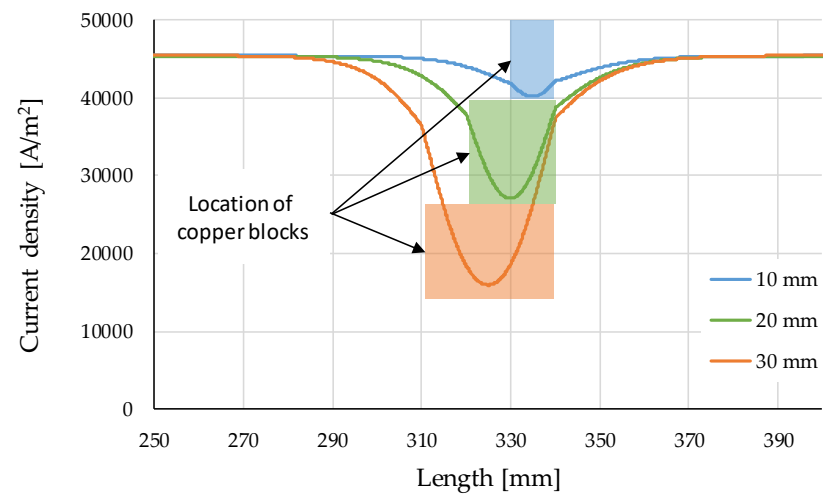

Fig. 6. Effect of the width of the copper blocks ( $w=10,20$ and $30 \mathrm{~mm}$ ) on the current density along the length of the centerline, as shown by the FEM simulation

It can be seen that the narrower the copper blocks are, the more homogeneous the current density is, and the smaller the length of the fiber bundle with inhomogeneous current density is.

We examined the area around the current electrodes separately. We performed the simulation with the three copper blocks' geometry presented earlier (Fig. 7 a), assuming a $20 \mathrm{~mm}$ long clamp with a pressure of $0.16 \mathrm{MPa}$ immediately after the copper blocks. To model this effect, we changed the material model in this section (Fig. 7 b). In reality, compressive forces would be applied with clamping jaws made of an insulating material, therefore we used transverse conductivity caused by higher pressures.

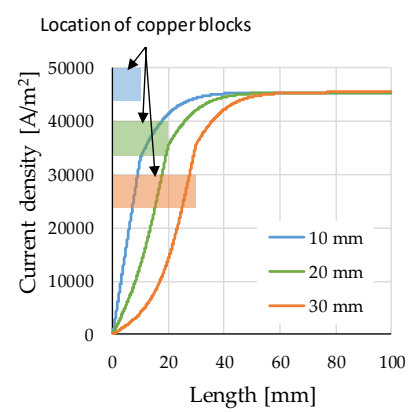

a)

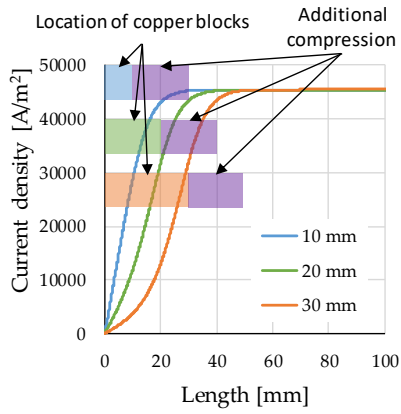

b)
Fig. 7. Effect of copper block width on the current density along the centerline (a), and its change when a $20 \mathrm{~mm}$ long clamp is used (b) based on FEM simulations

Comparing the two figures shows that due to the clamping force applied on a length increased by $20 \mathrm{~mm}$, the current density homogenizes faster and without a break in the curve after the copper blocks.

Based on the simulation results, the current density is not homogeneous around the copper blocks, which should be one 
of the conditions for the applicability of formula (1). We also showed that the narrower copper blocks used as the voltage electrode make resistance measurement more accurate, which is one of the basic requirements for self-sensing composites.

\section{Analysis of the effect of the media surrounding the fiber}

In the production of carbon fiber reinforced composites, the dry fiber bundles are first impregnated with a resin, which cures after the gel time. Therefore, we measured transverse resistance and its pressure dependence when the resin impregnated the bundle and also, when it was in a crosslinked matrix, in the arrangement shown in Fig. 1 b, similarly to the resistance measurements of the dry bundle (at the same pressure values). Before impregnation, we measured the resistance of the dry sample at the same pressures.

The difference in resistance measured on dry fiber bundles in the two measurement arrangements is due to the fact that, when the bundle is impregnated, it is necessary to lead the fiber bundles out; therefore their longitudinal resistance is added to the measurement results as an error. This constant error can be estimated from the longitudinal resistivity and the length of the fiber bundle, or approximated by the difference between the two arrangements. Subtracting the system resistance, we measured similar values in the two arrangements.

After the dry measurement, the tool cavity was filled with a resin not mixed with a crosslinker, and the resistance of the sample was measured (Fig. 8.). To do this, we removed the push-piece (Stage 1), then poured the resin on the surface of the carbon fiber bundle (Stage 2), and replaced the push-piece (Stage 3). With the lowest compressive force (0.01 MPa) previously applied, the two tools were compressed to fix the sample's shape, and the resin was pressed through the carbon fibers (Stage 4).

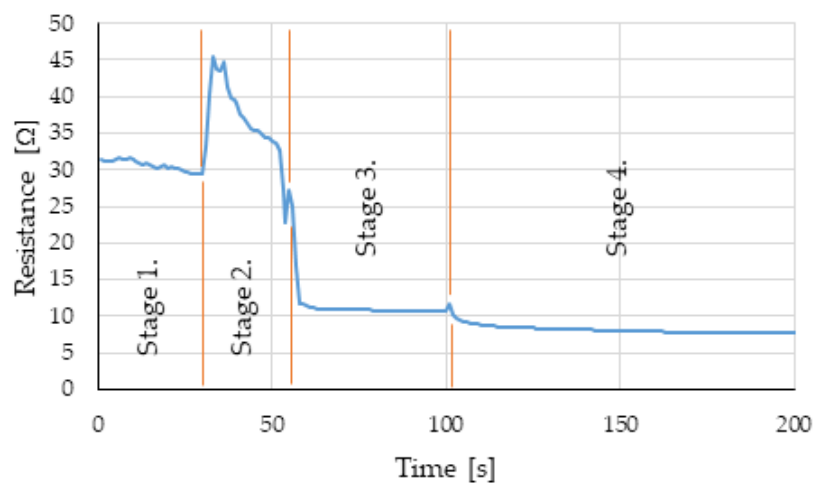

Fig. 8. Changes in the resistance of carbon fiber bundles during resin impregnation

Fig. 8 shows that the stabilized resistance in Stage 1 first increased in Stage 2 when the resin was poured in and then decreased as the elementary fibers moved away from each other and then were rearranged. In Stages 3 and 4, the resistance stabilizes again as the push-piece is applied and pressed: the excess resin is pushed out, the distance between the elementary fibers is reduced, and in some cases, new fiber-fiber contacts are formed.

After impregnation, we measured resistance at different pressures. Compared to dry fiber bundles, the resistance stabilized more slowly in this case, which was especially characteristic at low loading forces. It behaved differently even after the load was removed: the resistance slowly approached the no-load resistance value from below after a sudden increase. This can be explained with the resin's viscosity: due to the internal friction, the fibers move away from each other more slowly. Comparing the dry fiber and resin-impregnated fiber results, we found the expected difference: in the case of resin, we measured higher specific resistance values at different pressures, which can be explained with the insulating property of the resin (Fig. 9).

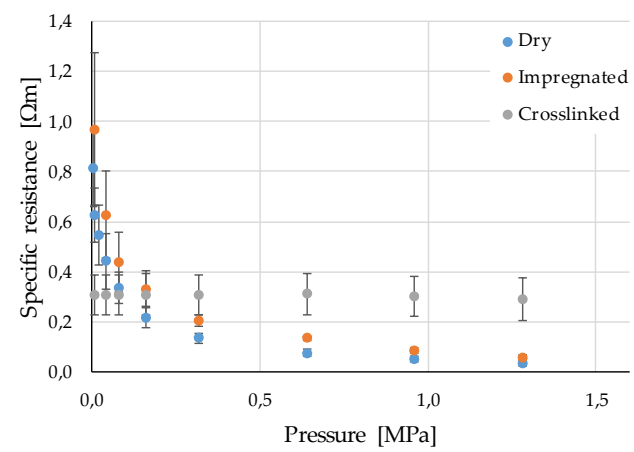

Fig. 9. Comparison of specific resistances of dry carbon fiber bundles, bundles in non-crosslinked resin and bundles in a crosslinked matrix as a function of pressure. The measurement points show the average of three specimens.

We also performed a series of experiments with a crosslinked matrix by preparing a new dry fiber bundle sample and then measuring the resistance at different pressures to determine the system's resistance. The cavity was then filled with the matrix material and crosslinked at room temperature. After crosslinking, resistance was measured again at different pressures (Fig. 9). The large standard deviation of the resistances of bundles in a crosslinked matrix is due to the fact that fiber arrangement was slightly different during the crosslinking of each specimen. The specific resistance of the specimens under pressure did not change significantly, as shown by the size of the constant mean and the constant error bar. The transverse specific resistance of the dry fiber bundles and fiber bundles in non-crosslinked resin depends significantly on the compressive force. On the other hand, in the case of a crosslinked matrix, the position of the elementary fibers, the number of fiber-to-fiber contacts, and thus the transverse specific resistance is fixed during crosslinking and do not change under pressure. This is why carbon fiber composites can be reliably used as sensors.

\section{E. Investigation of current distribution in resin- impregnated fiber bundles}

We showed that impregnation modifies the conductivity of the fiber bundles in a direction perpendicular to the fibers. In the following, we examine using the validated finite element model how the current distribution at the voltage electrodes changes due to impregnation. We compared the current drain effect of the copper blocks used for voltage measurement in the case of resin-impregnated and dry fiber bundles. We examined the environment of the voltage electrode "B" in Fig. 4, and constructed the model shown in Fig. 10. 


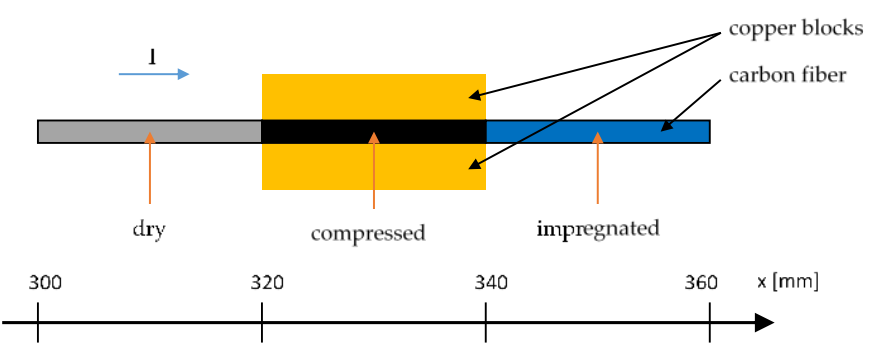

Fig. 10. Arrangement of the voltage measuring electrode environment in FEM for dry (gray) fiber and resin-impregnated (blue) fiber bundles.

The current flows in a dry, uncompressed fiber bundle $\left(\rho_{t}=\right.$ $0.54 \Omega \mathrm{m})$ to the section compressed by the copper blocks $\left(\rho_{t}=\right.$ $0.22 \Omega \mathrm{m}$ ). Following the copper blocks, the current continues to flow in the resin-impregnated fiber bundle, where we used a transverse specific resistance of $0.74 \Omega \mathrm{m}$ measured at a pressure of $0.01 \mathrm{MPa}$ (Fig. 9). Since the conductivity of the copper blocks is substantially higher than that of the carbon fibers, a portion of the current entering the section compressed by the copper blocks passes into the copper blocks and then returns to the fiber bundle on the opposite side of the copper blocks as shown in Fig. 4 d. Using our finite element model, we investigated this current drain effect in the geometry shown in Fig. 10 and plotted the current density distribution along the centerline of the fiber bundle as a function of location (Fig. 11).

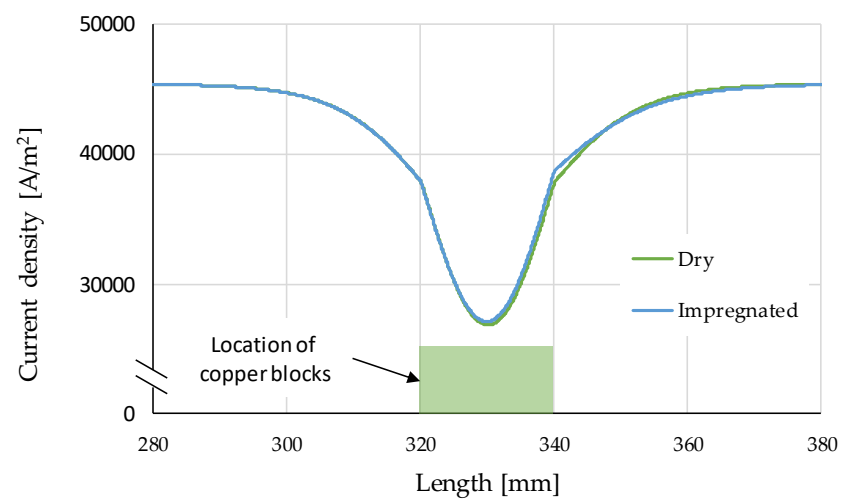

Fig. 11. Current density distribution along the centerline of the fiber bundle in the vicinity of the voltage electrode. Green line: for bundles dry on both sides Blue line: for bundles dry on one side and resinimpregnated on the other.

In the section in front of the electrode, the green and blue current density distribution curves run together since in this section, the fiber bundle was dry in both cases. The difference between the two curves is observed only in the section following the electrode, as here the green curve represents a dry fiber bundle, while the blue curve represents a bundle impregnated with resin. In the case of the impregnated bundle, the current drain effect is less pronounced; the current density inside the fiber bundle shows a $2 \%$ lower decrease than in the case of the dry fiber bundle. This is because the resin increases the specific resistance in a direction perpendicular to the fiber bundle, making it more difficult for the current to reach fibers not in direct contact with the copper blocks.

\section{CONCLUSION}

If reinforcing carbon fibers are to be used as a sensor, it is of paramount importance to accurately measure the longitudinal and transverse resistance and examine the current path within the carbon fiber bundle. In our paper, we measured the transverse resistance of carbon fiber bundles and created a finite element model based on the results. We showed that the current density distribution around the "current electrodes" is not uniform, preventing the accurate measurement of specific resistance. The inhomogeneity due to the anisotropic conductivity of the carbon fiber bundle must be taken into account in the multifunctional use of carbon fiber. In addition to the reinforcing role, its electrical conductivity is also used, for example, to measure deformation. In order for the current density to be homogeneously distributed over the entire crosssection, a uniform current input is required. Based on the finite element model, current density distribution can be made more uniform by using a smaller contact area and compressive force.

We also measured the carbon fiber bundle's transverse resistance at typical pressures and in circumstances (dry fiber bundles, fiber bundles in a non-crosslinked resin and fiber bundles in a crosslinked matrix) similar to the production of carbon fiber reinforced composites. We showed that carbon fiber bundles are sensitive to the compressive force when dry and impregnated with non-crosslinked resin, but this effect is negligible when the matrix is crosslinked. We explained our results mainly with the elementary fibers' physical position, that is, fiber-fiber contacts: in a dry fiber bundle, the fibers contact each other freely. Increasing the compression force would result in an increasing number of fiber-fiber contacts, reducing the transverse resistance significantly. Besides that, deformation of the fiber's contact area and the sizing would also decrease the transverse resistance marginally. In noncrosslinked resin, the resin impregnates the elementary fibers and isolates them from each other, but fiber-to-fiber connections can still be found due to imperfect impregnation. As the pressure is increased, the fibers are more compressed, and this reduces transverse resistance. When the fiber bundle is in a crosslinked matrix, the number of fiber-to-fiber contacts is fixed; it does not change under pressure.

We performed additional FEM simulations using the transverse specific resistance calculated from the resinimpregnated carbon fiber bundles' measured transverse resistance. We investigated the effect of the media around the voltage electrode on current density. We found that due to the higher transverse resistivity, the electrode's current-absorbing effect is less pronounced in resin-impregnated carbon fiber bundles.

With the fiber bundles' anisotropic specific resistance properties, the pressure dependence of the transverse specific resistance and the temperature dependence presented in our previous article [13] taken into account, a multifunctional carbon fiber reinforced composite can be created that can detect pressure and temperature during production. This allows product-specific and in-situ production control and quality assurance. Thus, it is essential that the current electrode and its environment are properly designed, because only then can a homogeneous and reproducible current density distribution be achieved. 


\section{ACKNOWLEDGMENT}

We would like to thank Bence Paréj for his measurements.

\section{REFERENCES}

[1] CCeV \& AVK. Global demand for carbon fiber from 2010 to 2022 (in 1,000 metric tons). Statista n.d. [Online] Avaiable:

https://www.statista.com/statistics/380538/projection-demand-forcarbon-fiber-globally (accessed September 24, 2019).

[2] N. Geier, J.P. Davim, and T. Szalay, "Advanced cutting tools and technologies for drilling carbon fibre reinforced polymer (CFRP) composites : A review," Compos. Part. A-Appl. Sci. Manuf., vol. 125, paper ID: 105552, 2019, doi:10.1016/j.compositesa.2019.105552.

[3] N. Geier, and C. Pereszlai, "Analysis of characteristics of surface roughness of machined CFRP composites," Period. Polytech. Mech. Eng., vol. 64. pp. 67-80, 2020,

doi:https://doi.org/10.3311/PPme.14436

[4] Z.A.M. Ishak, "Editorial corner - a personal view Recycled carbon fibre: A promising future," Express. Polym. Lett., vol. 13, paper ID: 1026, 2019, doi:10.3144/expresspolymlett.2019.89.

[5] M. Abedi, A. Avazpour, and B.A. Arand, "Hot carbon fiber acoustic vector sensor and flowmeter," IEEE Sens. J., vol 20, pp. 6670-6676, 2020, doi:10.1109/JSEN.2020.2976522.

[6] J. Reese, M. Vorhof, G. Hoffmann, K. Böhme, and C. Cherif, "Joule heating of dry textiles made of recycled carbon fibers and PA6 for the series production of thermoplastic composites," J. Eng. Fiber. Fabr., vol 15, pp 1-13, 2020, doi:10.1177/1558925020905828.

[7] B. Vermes, and T. Czigany, "Non-conventional deformations: Materials and actuation," Materials (Basel), vol 13, paper ID: 1383, 2020, doi:10.3390/ma13061383.

[8] N. Forintos, and T. Czigany, "Multifunctional application of carbon fiber reinforced polymer composites: Electrical properties of the reinforcing carbon fibers - A short review," Compos. Part. B-Eng., vol 162, pp 331-343, 2019, doi:10.1016/j.compositesb.2018.10.098.

[9] M.M.B. Hasan, A. Matthes, P. Schneider, and C. Cherif,

"Application of carbon filament (CF) for structural health monitoring of textile reinforced thermoplastic composites", Mater. Technol., vol 26, pp 128-134, 2011, doi:10.1179/175355511X13007211258881.

[10] C. Luan, X. Yao, H. Shen, and J. Fu, "Self-sensing of positionrelated loads in continuous carbon fibers-embedded 3D-printed polymer structures using electrical resistance measurement," Sensors, vol 18, paper ID: 994, 2018,. doi:10.3390/s18040994.

[11] B. Pinto, S. Kern, J.J. Ku-Herrera, J. Yasui, V. Saponara, and K.J. Loh, "A comparative study of a self strain-monitoring carbon nanotube film and carbon fibers under flexural loading by electrical resistance changes," J. Phys. Conf. Ser., vol 628, paper ID 12098, 2015, doi:10.1088/1742-6596/628/1/012098.

[12] A. A. Eddib, and D.D.L. Chung, "First report of capacitance-based self-sensing and in-plane electric permittivity of carbon fi ber polymer-matrix composite," Carbon, vol. 140, pp 413-427, 2018, doi:10.1016/j.carbon.2018.08.070.

[13] N. Forintos, and T. Czigany, "Reinforcing carbon fibers as sensors: the effect of temperature and humidity," Compos. Part. A-Appl. Sci. Manuf., vol. 131, paper ID: 105819, 2020, doi:10.1016/j.compositesa.2020.105819.

[14] N.D. Alexopoulos, C. Bartholome, P. Poulin, and Z. Marioli-Riga, "Structural health monitoring of glass fiber reinforced composites using embedded carbon nanotube (CNT) fibers," Compos. Sci. Technol., vol 70, pp 260-271, 2010,

doi:10.1016/j.compscitech.2009.10.017.

[15] V. Kumar, T. Yokozeki, T. Okada, Y. Hirano, T. Goto, T. Takahashi, and T. Ogasawara, "Effect of through-thickness electrical conductivity of CFRPs on lightning strike damages," Compos. Part. A-Appl. Sci. Manuf., vol. 114, pp 429-438, 2018, doi:10.1016/j.compositesa.2018.09.007.

[16] Y.X. Song, W.M. Xu, M.Z. Rong, and M.Q. Zhang, "A sunlight selfhealable fibrous flexible pressure sensor based on electrically conductive composite wool yarns," Express Polym. Lett., vol. 14, pp 1089-1104, 2020, doi: 10.3144/expresspolymlett.2020.88.

[17] C.N. Owston, "Electrical properties of single carbon fibres," J. Phys. D. Appl. Phys., vol 3, pp 1615-1626, 1970, doi:10.1088/0022$3727 / 3 / 11 / 309$.

[18] V. Šafářová, and J. Grégr, "Electrical conductivity measurement of fibers and yarns," in 7th Int. Conf. - TEXSCI 2010, Liberec, Czech Republic, 2010, p8.

[19] N. Athanasopoulos, and V. Kostopoulos, "Prediction and experimental validation of the electrical conductivity of dry carbon fiber unidirectional layers," Compos. Part. B-Eng., vol 42, pp 15781587, 2011, doi:10.1016/j.compositesb.2011.04.008.

[20] E.J. Zimney, G.H.B. Dommett, R.S. Ruoff, and D.A. Dikin, "Correction factors for 4-probe electrical measurements with finite size electrodes and material anisotropy: a finite element study," Meas. Sci. Technol., vol 18, pp 2067-2073, 2007, doi:10.1088/09570233/18/7/037.

[21] Q. Zhao, K. Zhang, S. Zhu, H. Xu, D. Cao, L. Zhao, R. Zhang, and W. Yin, "Review on the electrical resistance / conductivity of carbon fiber reinforced polymer," Appl. Sci., vol. 9, paper ID: 2390, 2019, doi:10.3390/app9112390.

[22] SGL Sigrafil carbon tow catalog n.d. [Online] Aviable: www.sglgroup.com (accessed December 10, 2019).

[23] Z.H. Xia, W.A. Curtin, "Modeling of mechanical damage detection in CFRPs via electrical resistance," Compos. Sci. Technol., vol 67, pp 1518-1529, 2007, doi: 0.1016/j.compscitech.2006.07.017.

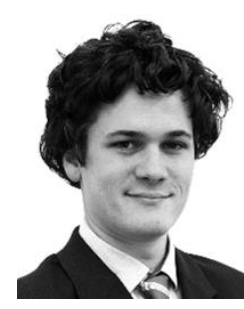

Norbert Forintos is currently a $\mathrm{PhD}$ student at the Department of Polymer Engineering at the Budapest University of Technology and Economics (BME). He received his M.Sc. degree at the Faculty of Mechanical Engineering, BME, in 2017.

His research now focuses on structural health monitoring of carbon fiber reinforced composite structures. He is the author of 3 paper and 5 proceedings.

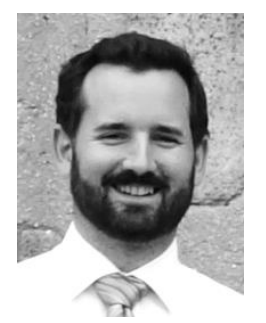

Tamas Sarkadi is currently an associate professor at the Department of Atomic Physics at Budapest University of Technology and Economics (BME) He received his master degree in engineering-physics in 2008. and his $\mathrm{PhD}$ in 2015. from BME. His research focuses on applied physics, optical measurement techniques, holography and quantum optics. He is the author of 18 paper and 5 proceedings.

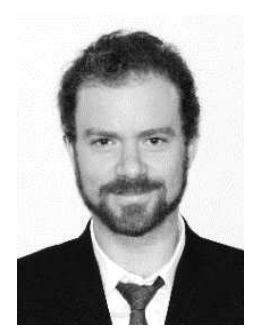

Csanád Örs Boros received his $\mathrm{PhD}$ degree in Physics at Budapest University of Technology and Economics in 2020. His research interest lies in the multiphysical simulation of complex microand nanostructures. His diploma work was multiphysical simulation of ship screw operation, especially modeling the effect of cavitation. $\mathrm{He}$ is the author of 4 paper and 3 proceedings.

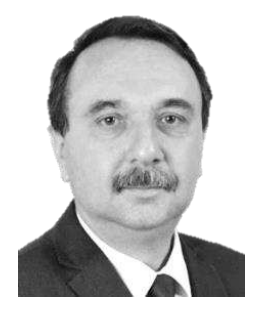

Prof. Tibor Czigany is currently a professor at the Department of Polymer Engineering at the Budapest University of Technology and Economics (BME). He received his mechanical engineering master's degree in 1988 and his $\mathrm{PhD}$ in 1997 from BME. He is a full member of the Hungarian Academy of Sciences (MTA). His research focuses on polymer engineering, composite materials and materials testing. $\mathrm{He}$ is the author of more than 250 papers and proceedings with more than 4600 independent citations. 